\title{
Accuracy of PET/CT for detection of infective endocarditis: Where are we now?
}

\author{
Jean-François Sarrazin, MD, FRCPC, FACC, FHRS, ${ }^{a}$ Mikaël Trottier, MD, ${ }^{b}$ and \\ Michel Tessier, $M D^{b}$ \\ a Division of Cardiology, Institut universitaire de cardiologie et de pneumologie de Québec, \\ Quebec, Canada \\ b Department of Nuclear Medicine, Institut universitaire de cardiologie et de pneumologie de \\ Québec, Quebec, Canada
}

Received Nov 3, 2017; accepted Nov 3, 2017

doi: $10.1007 / \mathrm{s} 12350-017-1126-2$

\section{See related article, pp. 922-935}

Today, patients with infective endocarditis (IE) or prosthetic valve endocarditis (PVE) still have a significant risk of morbidity and mortality. Early diagnosis is critical since delay in therapy has been associated with a poor outcome. ${ }^{1}$ Currently, we are still using the modified Duke criteria as the gold standard for the diagnosis of IE. $^{2}$ Cardiac imaging, mainly echocardiography, plays an essential role in the diagnosis and management of these patients. However, several patients are initially misclassified because of the limited early diagnostic accuracy of the modified Duke criteria. In addition, echocardiography has some limitations, since a negative study does not rule out IE and a false positive diagnosis can occur in up to $15 \%$ of patients with non-infective vegetations. ${ }^{3}$ For these reasons, other imaging techniques have clearly some potential benefits.

18F-FDG PET/CT has evolved over the past few years as an additional diagnostic tool in the management of cardiovascular implantable electronic device (CIED) infection and PVE. Indeed, it has been incorporated in the 2015 guidelines for the management of IE from the European Society of Cardiology when the diagnosis is still possible or has been rejected but a high degree of

Reprint requests: Jean-François Sarrazin, MD, FRCPC, FACC, FHRS, Division of Cardiology, Institut universitaire de cardiologie et de pneumologie de Québec, 2725, chemin Sainte-Foy, Quebec, G1V 4G5, Canada; jean-francois.sarrazin@criucpq.ulaval.ca

J Nucl Cardiol 2019;26:936-8.

$1071-3581 / \$ 34.00$

Copyright (c) 2017 American Society of Nuclear Cardiology. suspicion still remain. ${ }^{4}$ The combination of radionuclide imaging to low-dose CT scan has been a great advancement. It has several advantages, including an excellent spatial resolution, a short acquisition time, a high sensitivity for the detection of hypermetabolic activity, and the ability of detecting peripheral events, defining the extent of infection or identifying other sources of infection. ${ }^{5} \mathrm{~A}$ previous study from Saby et al has demonstrated an additional benefit of using abnormal 18F-FDG uptake in the detection of PVE. ${ }^{6}$ It can improve the sensitivity of the modified Duke criteria and reduce the number of misdiagnosed cases. It has also been shown that the result of the PET/CT could lead to modification of the treatment in as much as one third of patients. ${ }^{7}$ Till now, studies have been limited by a small number of patients.

In this issue of the Journal, Mahmood et al reported a meta-analysis on the use of 18F-FDG PET/CT for the diagnosis of IE. ${ }^{8}$ The overall sensitivity was $76.8 \%$ with a specificity of $77.9 \%$. The sensitivity was improved to $80.5 \%$ in patients with PVE. One of the additional benefits of PET/CT was the possibility to identify an extra-cardiac source of infection in $17 \%$ of patients. This meta-analysis included 13 studies published between 2013 and 2017, for a total of 537 patients. This represents the largest pool of patients so far. However, they were also able to demonstrate a significant heterogeneity between the studies. Some studies included both native IE and PVE, while other only PVE or also CIED infections. There was no standardized protocol, and intravenous heparin was used in only three studies out of nine with a reported PET/CT protocol. They also showed that more recent studies had a higher pooled sensitivity.

The accuracy of this test appears lower than initially expected. There are potentially several reasons to explain this result. The first issue is the lack of a standardized protocol between centers and across the 
different studies. As reported in this manuscript, there are significant variations between the different PET/CT protocols in the literature, since there is still no consensus on the best patient preparation before and during the study. Some authors advocate the importance of a very long fasting period, up to 18 hours before the injection of $18-\mathrm{FDG},{ }^{9}$ while others insist on combining all methods, i.e., the use of a low carbohydrate / high fat diet (Atkins diet) for at least 24 hours but up to 48 hours, a period of fast between 8 and 12 hours and intravenous heparin pre-administration. ${ }^{10} \mathrm{We}$ definitely think that a rigorous protocol is critical to completely suppress physiological myocardial uptake in order to obtain a better discrimination between normal and abnormal 18F-FDG activity within the myocardium. An adequate PET/CT protocol should include a prolonged fasting period of 8 to 12 hours, the use of the Atkins diet, controlling blood glucose, and the administration of $50 \mathrm{IU} / \mathrm{kg}$ intravenous heparin 15 minutes prior to injection of $18 \mathrm{~F}-\mathrm{FDG}$. In addition, there are no validated specific diagnostic criteria for the interpretation of PET/ CT in the evaluation of IE. Diagnosis is mainly based on a qualitative value or visual inspection of abnormal $18 \mathrm{~F}$ FDG uptake and its intensity (SUV max). Analysis of both attenuation-corrected and non-attenuation-corrected images need to be reviewed in order to recognize potential artifacts from the prosthesis. This is extremely important, and it was not properly addressed in the article. It could explain some of the heterogeneity between studies. Ignoring the non-attenuation-corrected images can contribute to a higher number of false positive studies and can decrease the specificity in patients with prostheses and heavily calcified valves. Also, different groups have been using different semiquantitative methods. A semi-quantitative count ratio (SQR) on non-attenuation-corrected images is very important in order to compare to a reference organ (either the lung or the liver parenchyma). At this time, a universal method for both SUV and SQR measurements is lacking (region of interest drawing, SUV max or SUV mean, organ of reference). ${ }^{11}$ In addition, it is worth noting that most patients in these studies were already receiving antibiotics (75\%), and that in some studies, patients have been already treated for more than 1 week. Prolonged antibiotic therapy prior to radionuclide imaging can clearly have a direct impact on the results of the test by decreasing or suppressing the area of infectious activity. ${ }^{12}$ This can lead to false negative studies and affect the sensitivity of the test. Unfortunately, PET/CT is not available everywhere. Finally, we should recognize that in some patients, especially those with mechanical valves, chronic inflammation may cause non-specific, albeit fairly high uptake, sometimes persisting for years after the surgery, and this can contribute to some of the false positives and lower specificity. Other diagnostic methods such as radiolabelled leukocytes should also be explored in these patients.

In conclusion, in order to incorporate PET/CT imaging more regularly in the management of patients with IE or PVE, larger studies with standardized protocols are required. Mahmood et al rightly mentioned that the accuracy of the test could possibly vary according to the type of pathogen, and this could also be addressed in future studies. More studies are also required for the early postoperative management of patients with IE since PET/CT cannot differentiate easily between an infection and the residual inflammation. Such studies should look at PET/CT combined to radiolabelled leukocytes since it is more specific. However, for the moment, the published meta-analysis by Mahmood et al offers a more global view on this topic.

\section{Disclosures}

The authors have nothing to declare.

\section{References}

1. Dickerman SA, Abrutyn E, Barsic B, Bouza E, Cecchi E, Moreno $A$, et al. The relationship between the initiation of antimicrobial therapy and the incidence of stroke in infective endocarditis: An analysis from the ICE prospective cohort study (ICE-PCS). Am Heart J 2007;154:1086-94.

2. Li JS, Sexton DJ, Mick N, Nettles R, Fowler VG Jr, Ryan T, et al. Proposed modifications to the Duke criteria for the diagnosis of infective endocarditis. Clin Infect Dis 2000;30:633-8.

3. Habib G, Derumeaux G, Avierinos JF, Casalta JP, Jamal F, Volot $\mathrm{F}$, et al. Value and limitations of the Duke criteria for the diagnosis of infective endocarditis. J Am Coll Cardiol 1999;33:2023-9.

4. Habib G, Lancelloti P, Antunes MJ, Bongiorni MG, Casalta JP, Del Zotti F, et al. 2015 ESC Guidelines for the management of infective endocarditis: The task force for the management of infective endocarditis of the European society of cardiology (ESC). Endorsed by: European association for cardio-thoracic surgery (EACTS), the European association of nuclear medicine (EAN. Eur Heart J 2015;36:3075-128.

5. Sarrazin JF, Philippon F, Trottier M, Tessier M. Role of radionuclide imaging for diagnosis of device and prosthetic valve infections. World J Cardiol 2016;8:534-46.

6. Saby L, Laas O, Habib G, Cammilleri S, Mancini J, Tessonnier L, et al. Positron emission tomography/computed tomography for diagnosis of prosthetic valve endocarditis: Increased valvular 18Ffluorodeoxyglucose uptake as a novel major criterion. J Am Coll Cardiol 2013;61:2374-92.

7. Van Riet J, Hill EE, Gheysens O, Dymarkowski S, Herregods MC, Herijgers $\mathrm{P}$, et al. 18F-FDG PET/CT for early detection of embolism and metastatic infection in patients with infective endocarditis. Eur J Nucl Med Mol Imaging 2010;37:1189-97. 
8. Mahmood M, Kendi A, Ajmal S, Farid S, O'Horo J, Chareonthaitawee $\mathrm{P}$, et al. Meta-analysis of $18 \mathrm{~F}-\mathrm{FDG} \mathrm{PET} / \mathrm{CT}$ in the diagnosis of infective endocarditis. J Nucl Cardiol 2017. https://doi.org/10.1007/s12350-017-1092-8.

9. Langah R, Spicer K, Gebregziabher M, Gordon L. Effectiveness of prolonged fasting 18f-FDG PET-CT in the detection of cardiac sarcoidosis. J Nucl Cardiol 2009;16:801-10.

10. Scholtens AM, Verberne HJ, Budde RP, Lam MG. Additional heparin preadministration improves cardiac glucose metabolism suppression over low-carbohydrate diet alone in 18F-FDG PET imaging. J Nucl Med 2016;57:568-73.

11. Scholtens AM, Swart LE, Kolste HJT, Budde RPJ, Lam MGEH, Verberne HJ. Standardized uptake values in FDG PET/CT for prosthetic heart valve endocarditis: a call for standardization. $\mathrm{J}$ Nucl Cardiol 2017. https://doi.org/10.1007/s12350-017-0932-x.

12. Scholtens AM, van Aarnhem EE, Budde RP. Effect of antibiotics on FDG-PET/CT imaging of prosthetic heart valve endocarditis. Eur Heart J Cardiovasc Imaging 2015;16:1223. 